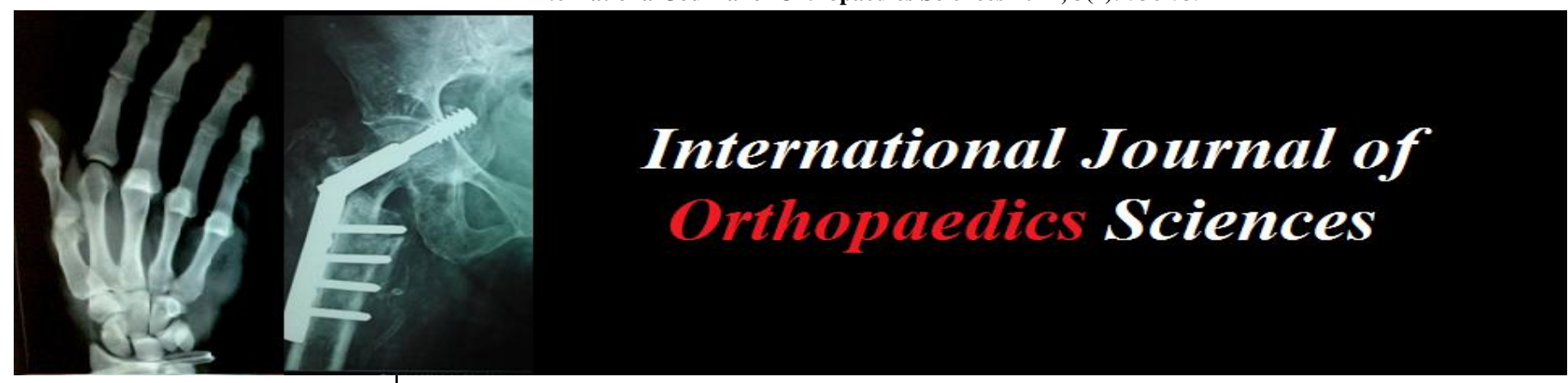

ISSN: $2395-1958$

IJOS 2017; 3(4): 536-539

(C) 2017 IJOS

www.orthopaper.com

Received: 12-08-2017

Accepted: 13-09-2017

\section{Sinisa Ducic}

MD, PhD, Pediatric orthopedic surgeon, Clinical Assistant,

University Children's Hospital

Belgrade, Serbia, Medical faculty

of Belgrade, Serbia

\section{Nikola Bojovic}

MD, Pediatric orthopedic surgeon, Clinic for Pediatric Surgery and Orthopedic Nis,

Clinical Center Nis, Serbia

\section{Maja Raicevic}

MD, Pediatric surgery resident, Clinic for Pediatric Surgery and Orthopedic Nis, Clinical Center Nis, Serbia

\section{Vladimir Radlovic}

MD, Pediatric surgeon,

University Children's Hospital

Belgrade, Serbia

\section{Goran Djuricic}

MD, Radiologist, University Children's Hospital Belgrade,

Serbia

Bojan Bukva

MD, PhD, Pediatric orthopedic surgeon, University Children's

Hospital Belgrade, Serbia
Correspondence

Nikola Bojovic

MD, Pediatric orthopedic surgeon, Clinic for Pediatric Surgery and Orthopedic Nis, Clinical Center Nis, Serbia

\section{Late presentation of congenital pseudarthrosis of the clavicle}

\author{
Sinisa Ducic, Nikola Bojovic, Maja Raicevic, Vladimir Radlovic, Goran \\ Djuricic and Bojan Bukva
}

DOI: $\underline{\text { https://doi.org/10.22271/ortho.2017.v3.i4h.72 }}$

\section{Abstract}

Congenital pseudarthrosis of the clavicle is a rare clinical entity, first described in 1910. We report on a 15 year old girl, who presented with a painless lump over mid-portion of right clavicle at her routine newborn examination, which was subsequently diagnosed as a congenital pseudarthrosis when she got into adolescence period. Here we explore its pathogenesis, elaborate on its diagnoses and differential diagnoses in paediatric patients, and comment on its distinct treatment.

Keywords: Congenital pseudarthrosis of clavicle, bone graft, clavicle, congenital, pseudarthrosis, shoulder girdle, surgical procedures

\section{Introduction}

Congenital pseudarthrosis of the clavicle is a rare benign defect of the formation of the clavicle, first presented in the literature by Fitzwilliams in 1910 and St. Pierre in 1930, respectively, each described one case ${ }^{[6]}$. The lesion presents as a nontender swelling situated slightly lateral to the mid-clavicle, having a variable degree of painless motion at that site and usually noticed at birth or occasionally in infancy and early childhood, but often diagnosed late in childhood ${ }^{[13]}$. Spontaneous union of the pseudarthrosis does not occur. The pseudoarthrosis increases in size over a period of years, giving rise to a progressive deformity, often encroaching on the skin, and frequently necessitating surgical treatment. The right clavicle is most commonly affected, although bilateral involvement has been reported in approximately $10 \%$ of cases ${ }^{[1]}$, and rare examples of isolated left-sided involvement have been documented ${ }^{[12]}$. Congenital pseudarthrosis of the clavicle is different from obstetric fracture, post-traumatic non-union, cleidocranial dysostosis, or neurofibromatosis ${ }^{[3]}$.

Our aim is to report a case of congenital pseudoarthrosis of the right clavicle in an adolescent, which is atypical age group for the first diagnose of this condition. We decided to resect pseudoarthrosis, do the autologous bone grafting and fixation with intramedullary " $\mathrm{K}$ " wire, plate and two screws. Union was achieved after 3 months.

\section{Case presentation}

A 15-year-old girl presented with a prominence of about $3 \mathrm{~cm}$ in size between the middle and distal third of the right clavicle (Fig. 1).

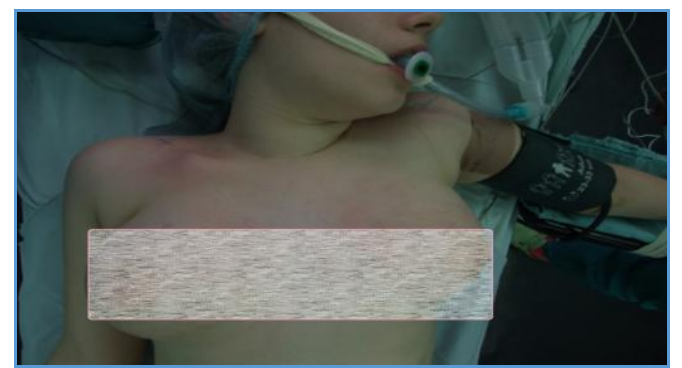

Fig 1: Clinical appearance shows prominence in the midpoint of the right clavicle $\sim 536 \sim$ 
The lump was present at birth. There was no history of a difficult delivery or injury. Family history was negative for similar anomalies, and there was no history of teratogenic agents during pregnancy nor other abnormalities of pregnancy or delivery. The baby got APGAR score 10 on delivery. As a baby she had regular pediatric examinations in the first week, the first month etc. Doctors understood the clavicle lump as a fracture due to delivery and explained to parents the course of fracture healing. In next 15 years girl did not have any pain, any discomfort, malaise in clavicle or right arm regions. Also she did not complain to any right shoulder movement limitations. We need to emphasize that throughout the years the girl was plump and clavicle lump was discreet. Few months after $15^{\text {th }}$ birthday she started to feel discomfort within the full range of right shoulder movements. Sometimes it was accompanied by pain. After menarche she began to lose weight and clavicle lump became more obvious. Her pediatrician sent her firstly to physiatrist who tried to treat her symptoms for two weeks. Because of no improvement she got referral to pediatric orthopedic surgeon.

Physical examination revealed a soft, non-tender mass in the middle part of clavicle diaphysis. The size of the mass increased with movements of the right shoulder and with abduction and elevation of the right arm, causing mild pain. Skin over the lump was normal.

Anamnesis, clinical findings and plain radiographs of the both shoulders and clavicles set a suspicion of congenital pseudarthrosis of the right clavicle. Radiographs showed an enlargement in the acromial and sternal borders of the clavicle (Fig.2)

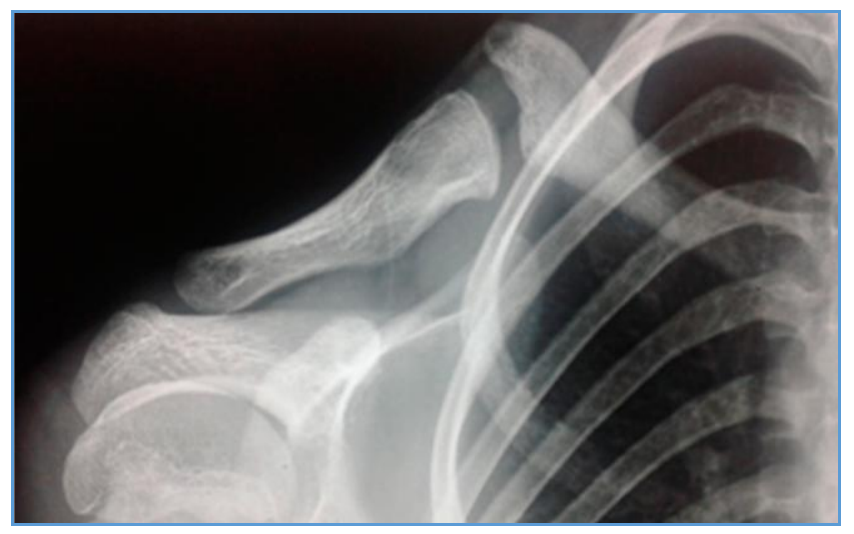

Fig 2: Pseudoarthrosis of clavicle with enlargement of it's midportion

Because of inadequate age for previous initial diagnosis and the rarity of disorder we decided to do the computed tomography (CT) of right clavicle and shoulder (Fig 3).

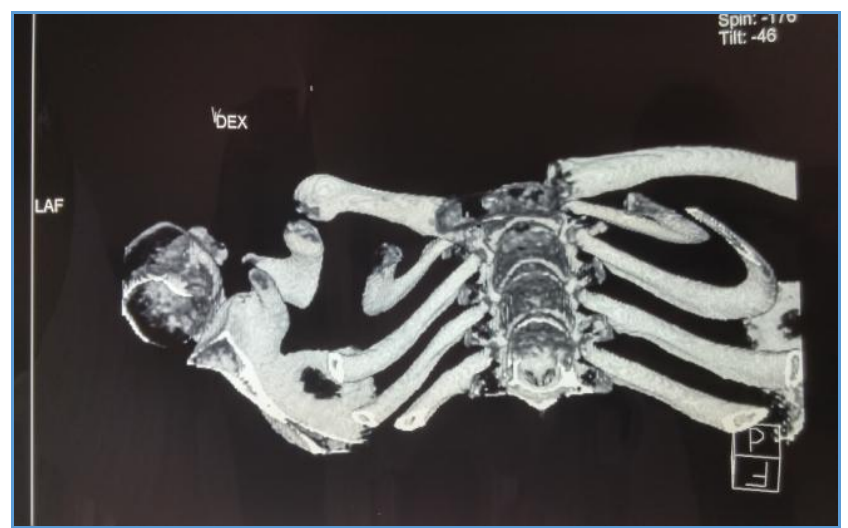

We confirmed the diagnosis and also determined the extent of the fibrous union and the location of the great vessels. Further observation was no appropriate and there was no known nonoperative techniques to achieve union.

Surgical treatment was offered to correct the unacceptable appearance and the functional problems in the right shoulder and arm. The patient was in supine position with raise right shoulder. A $6-\mathrm{cm}$ horizontal incision was made above the clavicle prominence parallel to clavicle, followed by a sharp dissection through the subcutaneous tissues to expose the clavicle. All fibrous and cartilaginous tissue was removed (app. $3 \times 2 \mathrm{~cm}$ ) from the site of the pseudarthrosis until healthy osseous tissue was exposed (Fig. 4).

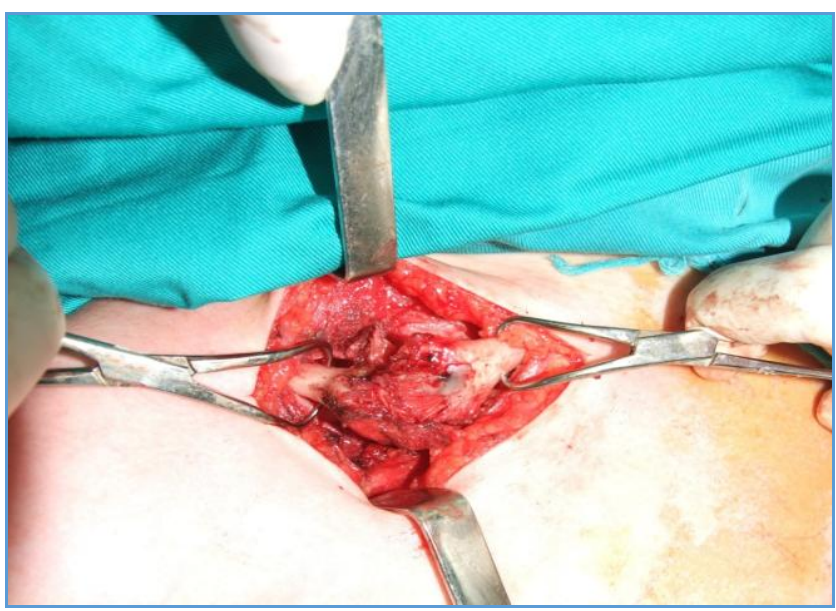

Fig 4: Fibro cartilaginous tissue in the midportion of clavicle

Interfragmental gap was app. $4 \mathrm{~cm}$ (Fig. 5). We sent two removed parts app. 1 x $3 \mathrm{~cm}$ to pathohistology exam. Pathohistological finding: fibrocartilaginous and osteochondral proliferation at both specimens which correspond to pseudoarthrosis of clavicle. The length of the clavicle diaphysis was reestablished with bone grafting. We took bone autograft from ipsilateral iliac bone.

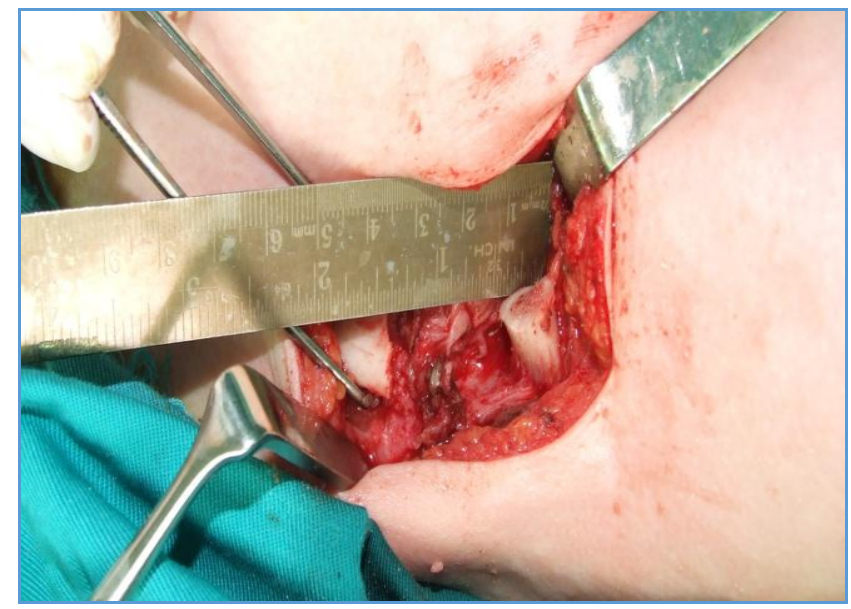

Fig 5: Interfragmental gap between two healthy clavicle's portion

The clavicular segments were fixed within combination of anterograde launched intramedullary " $\mathrm{K}$ " wire and plate and 2 screws (Fig. 6). Postoperatively a collar and cuff sling was worn for 3 weeks. The first signs of healing appeared 4 weeks after surgery and complete union achieved at 3 months.

Fig 3: MSCT scan of right clavicle 


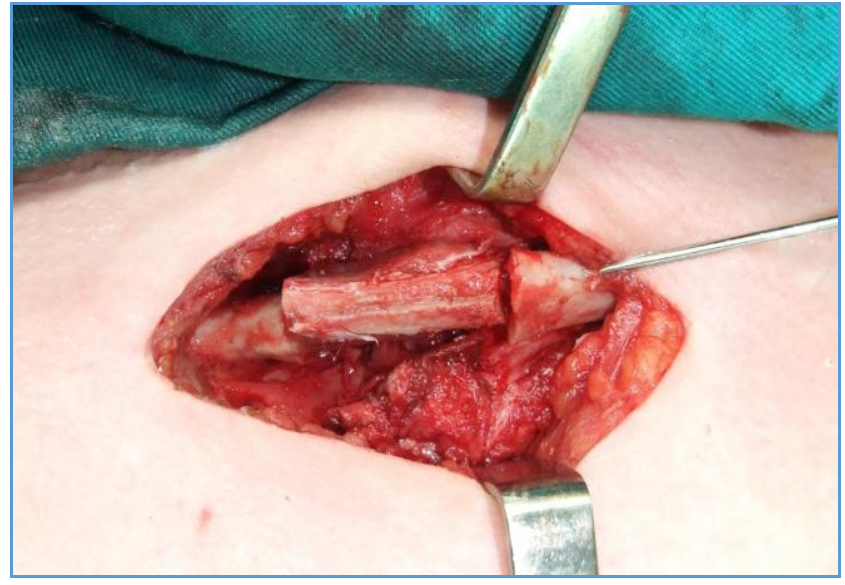

Fig 6: Iliac crest bone autograft

The final radiograph taken 1 year postoperatively was satisfactory, even though the right clavicle was $1 \mathrm{~cm}$ shorter than the left (Fig. 7). The patient had full range of movement of the right shoulder and arm, and was pain-free. The appearance of the shoulder girdle and upper arm was normal.

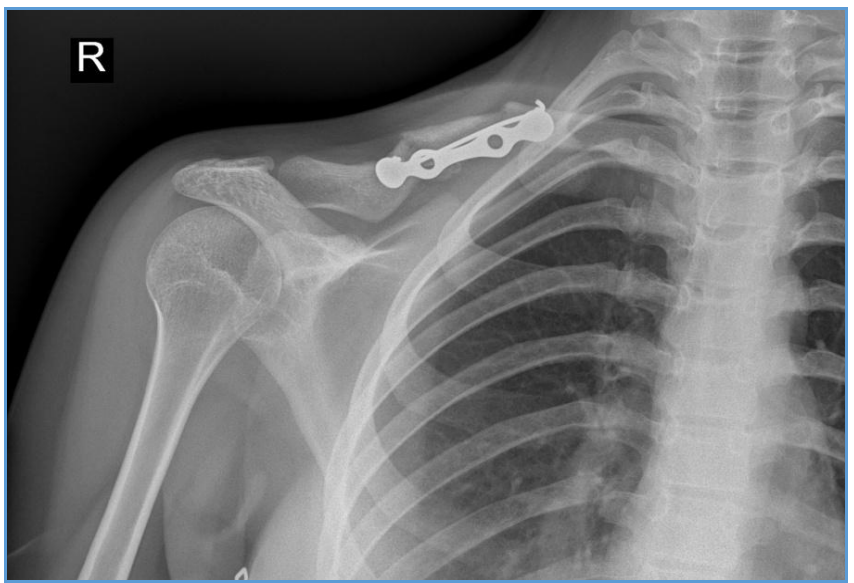

Fig 7: Post-op radiograph shows dual fixation

After 12 months follow up the patient came back for osteosynthetic material removal. In this second operation both plate and " $\mathrm{K}$ " wire were removed. The bone was completely united either in the radiography and at operation. On the Xray that was taken after this surgery (Fig.8), right clavicle was only $5 \mathrm{~mm}$ shorter than left clavicle. Last photography (Fig.9) shows appearance of the patient after plate removal.

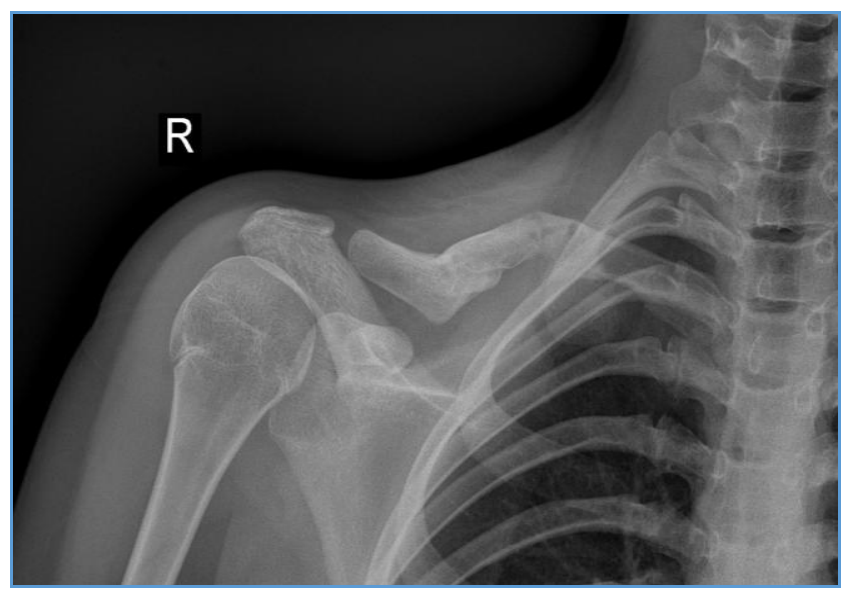

Fig 8: Final radiograph after alanthesis removal

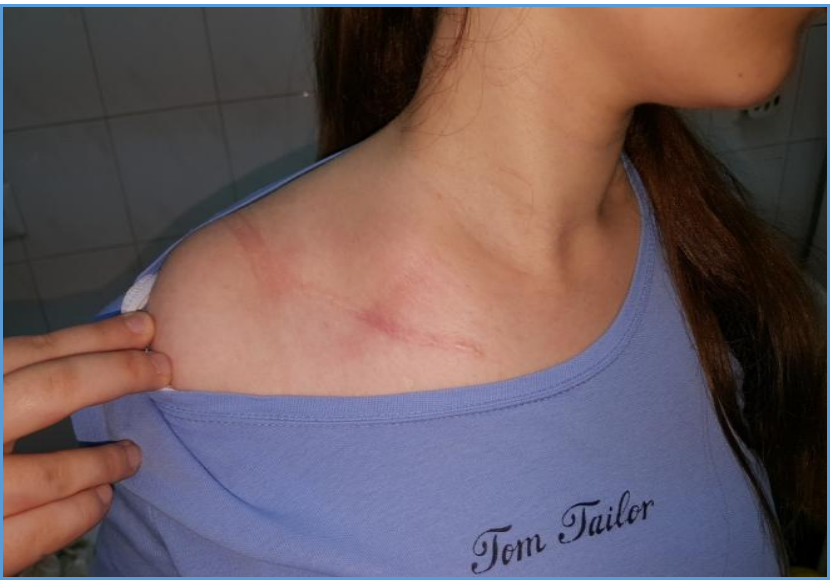

Fig 9: Appearance of the patient after alanthesis removal

\section{Discussion}

Congenital pseudarthrosis of the clavicle typically presents in infancy or in early childhood, with a painless prominence and hypermobile segment over the middle third of the clavicle mostly found in girls ${ }^{[14,7]}$. The cosmetic deformity tends to be slowly progressive, with an increase in noticeable prominence over the pseudarthrosis and foreshortening and dropping of the shoulder girdle over time. Sometimes can become painful with certain overhead activities, or with direct compression or palpation. Shoulder motion is typically regarded as normal, and significant functional disability is unusual. Several cases of later development of thoracic outlet compression syndrome have been reported in association with congenital pseudarthrosis of the clavicle ${ }^{[8,5]}$.

A genetic basis for congenital pseudarthrosis of the clavicle and possible an autosomal dominant type of transmission is unclear but suggested and illustrated by Gibson and Carrol ${ }^{[9]}$. The development of the clavicle from two separate centers and failure of their coalescence can be responsible for the development of pseudarthrosis ${ }^{[2]}$. Currently most authors believe that the condition is caused by extrinsic pressure exerted on the budding clavicle by the adjacent pulsatile right subclavian artery directly under the area of the defect and normally situated at a higher location compared to the left side ${ }^{[11]}$. Rare cases of left-side involvement have been associated with dextrocardia ${ }^{[1]}$, and bilateral cases have been associated with cervical ribs.

Radiological appearances show a definite separation of clavicle in its midportion, with the medial aspect lying superior to the lateral aspect, with no evidence of callus formation or reactive bones.

Indications for operative management for many authors have included progressive pain or other symptoms, unacceptable cosmetic deformity at the site of the pseudarthrosis, functional limitations and, at later stages, onset of thoracic outlet compression syndrome._Some authors discourage surgical treatment when patients are asymptomatic and have no functional disability. Also, because of the risk of nonunion, clavicle shortening and surgical scar. The true natural progression of untreated cases is unknown._The optimal age for surgery is controversial. Most specialists recommend delaying surgery until the patient is approximately three to six years of age ${ }^{[2,10]}$. Others recommend early surgical treatment during infancy. Manuscripts reported surgery time is from 18 months till adulthood.

Many procedures have been carried out for the surgical treatment of this condition. There are no big series and long follow-up. Grogan et al. ${ }^{[10]}$ reported excellent results in eight 
children (from 7 months to 6 years) in whom the fibrous pseudarthrosis was resected early, carefully preserving the continuity of the periosteal sleeve and approximating the bone ends without grafting. External fixation after pseudoarthrosis excision without bone grafting has been described with good result, smaller scar and avoidance of the second operation for hardware removal ${ }^{[4]}$.

After the age of 8 years, it appears to be more difficult to obtain fusion, and an alternative to bone grafting is excision of the prominent lateral end of the sternal half of the clavicle. The most surgical methods consists of resection of the pseudarthrosis followed by bone grafting and internal fixation of the bone ends. Others have suggested excision of the pseudarthrosis and grafting without using any type of fixation. Fixation with K-wire and plates with good results have been reported. Most authors prefer iliac crest bone graft to achieve union and maintain clavicle length but local bone graft or no bone graft are another options.

\section{Conclusion}

In our case, we described a patient with CPC of the right clavicle with late onset of complaints, nearly after 15 years of age. Surgery was carried out, the area of pseudarthrosis being excised. A iliac crest bone graft was laid across the defective area and fixed to both clavicle fragments with $\mathrm{K}$-wire, plate and two screws. Solid union followed. We recommend surgical treatment of congenital pseudarthrosis only if there is increasing pain with functional limitations or unacceptable cosmetic deformity regardless of age.

\section{References}

1. Ahmadi B, Steel HH. Congenital pseudoarthrosis of clavicle. Clin Orthop 1977; 126:130-134.

2. Alldred AJ. Congenital pseudarthrosis of the clavicle. J Bone Joint Surg. 1963; 45B:312-319.

3. Beals RK, Sauser DD. Nontraumatic disorders of the clavicle. J Am Acad Orthop Surg. 2006; 14(4):205-14.

4. Beslikas TA, Dadoukis DJ, Gigis IP, Nenopoulos SP, Christoforides JE. Congenital pseudarthrosis of the clavicle: a case report. Journal of Orthopaedic Surgery. 2007; 15(1):87-90.

5. Currarino G, Herring JA. Congenital pseudarthrosis of the clavicle. Pediatr Radiol. 2009; 39(12):1343-9.

6. Fitzwilliams DCL. Hereditary cranio-cleido-dysostosis. Lancet 2 1910, 1466-1475.

7. Galanopoulos I, Ashwood N, Garlapati AK, Fogg Q. Congenital pseudarthrosis of clavicle: illustrated operative technique andhistological findings. BMJ Case Rep 2012.

8. Garnier D, Chevalier J, Ducasse E. Arterial complications of thoracic outlet syndrome and pseudarthrosis of the clavicle: three patients. J Mal Vasc. 2003; 28(2):79-84.

9. Gibson DA, Carroll N. Congenital pseudoarthrosis of clavicle. J Bone Joint Surg (Br) 1970; 52:629-643.

10. Grogan DP, Love SM, Guideria KJ, Ogden JA. Operative treatment of congenital pseudarthrosis of the clavicle. $\mathrm{J}$ Pediatr Orthop. 1991; 11:176-180.

11. Lloyd-Roberts GC, Apley AG, Owen R. Reflection upon the etiology of congenital pseudoarthrosis of the clavicle. J Bone Joint Surg (Br). 1975; 57:24-29.

12. Sakkers RJ, Tjin a Ton E, Bos CF. Left-sided congenital pseudarthrosis of the clavicula. J Pediatr Orthop B. 1999; $8(1): 45-7$.

13. Sales de Gauzy J, Baunin C, Puget C. Congenital pseudarthrosis of the clavicle and thoracic outlet syndrome in adolescence. J Pediatr Orthop B. 1999; 8(4):299-301.

14. Tony HT Sung, Eric MW Man, Augustine TS Chan, Lee WK. Congenital pseudarthrosis of the clavicle: a rare and challenging diagnosis. Hong Kong Med J. 2013; 19(3):265-7. 